\title{
May Cause Dizziness: Applying the Simulator Sickness Questionnaire to Handheld Projector Interaction
}

\author{
Bonifaz Kaufmann \\ Alpen-Adria-Universität Klagenfurt \\ Universitätsstraße 65-67 \\ 9020 Klagenfurt, Austria \\ bonifaz.kaufmann@aau.at
}

\author{
Philip Kozeny \\ Alpen-Adria-Universität Klagenfurt \\ Universitätsstraße 65-67 \\ 9020 Klagenfurt, Austria \\ pkozeny@edu.aau.at
}

\author{
Stefan Schaller \\ Alpen-Adria-Universität Klagenfurt \\ Universitätsstraße 65-67 \\ 9020 Klagenfurt, Austria \\ sschalle@edu.aau.at
}

\author{
John N.A. Brown \\ Alpen-Adria-Universität Klagenfurt \\ Universitätsstraße 65-67 \\ 9020 Klagenfurt, Austria \\ jna.brown@aau.at
}

\author{
Martin Hitz \\ Alpen-Adria-Universität Klagenfurt \\ Universitätsstraße 65-67 \\ 9020 Klagenfurt, Austria \\ martin.hitz@aau.at
}

\begin{abstract}
Previous user studies have suggested the occurrence of symptoms of motion or simulator sickness among active spectators of handheld projector interaction. Using the well-established Simulator Sickness Questionnaire proposed by Kennedy et al. in 1993, we asked twenty-six participants if they had any indication of such symptoms after they watched a demonstration of handheld projector interaction for about half an hour. We show that handheld projector sickness can occur in rare situations, but overall it is not a substantive problem.
\end{abstract}

Handheld projector, motion sickness, simulator sickness, peephole pointing, user experience.

\section{INTRODUCTION}

Smartphone displays have been growing in size for several years. However, above a certain display size, a smartphone starts to become bulky resulting in a loss of its popular feature; mobility. One way to increase display size without giving up mobility is to integrate projection technology into smartphones. Besides a much larger display, handheld projector devices allow for new interaction mechanisms. Beardsley et al. (2005) were among the first who investigated interactive handheld projectors. Since then researchers explored this new interaction technology in multi-user scenarios (Cao et al. (2007)), games (Willis et al. (2011)) or even as an interactive phone call solution (Winkler et al. (2011)).

As with any new interactive technology, little is known about human factors that are involved when dealing with interactive handheld projectors. Willis et al. (2011) studied a new metaphor which they call MotionBeam, in which a virtual character can be controlled by moving the handheld projector that displays the character.
The researchers mention that some participants of their study had issues following the moving projected display because of symptoms normally related to motion and simulator sickness. Similar indications have been found during a user study that investigated peephole pointing with a handheld projector (Kaufmann and Ahlström (2012)).

In peephole pointing and also in MotionBeam interaction the projected display undergoes plenty of movement. Since, interactive handheld projectors might often be used in collaborative settings, and since various mechanisms in handheld projector interaction require a moving display, it is vital to raise the question if looking at a moving projected display will cause symptomatic reactions akin to motion or simulator sickness. Therefore, we conducted a user study with twenty-six participants using an established questionnaire to quantify the severity level of sickness symptoms resulting from handheld projector interaction. 


\section{BACKGROUND}

Simulator sickness was initially reported by Havron and Butler (NAVTRADEBCEN 1915-00-1 (1957)) when they evaluated a helicopter training simulation. Simulator sickness has been identified as a special form of motion sickness that does not rely on body movement but on vection, the effect that causes a person to feel self-motion when a large area of the visual field moves. Disparities between visual and vestibular information about motion can lead to sickness symptoms like nausea, vertigo, dizziness, eyestrain and several other symptoms that have been classified as simulator sickness symptoms (Kennedy et al. (1993)).

For measuring simulator sickness, Kennedy et al. (1993) developed the Simulator Sickness Questionnaire (SSQ). The SSQ is a symptom checklist that includes sixteen symptoms (e.g. dizzy, headache, eyestrain, etc.) related to simulator sickness. A participant rates each symptom according to four levels of severity: none, slight, moderate and severe. The result of the SSQ is used to calculate sub scores for nausea, oculomotor, disorientation and a total severity score that describes the overall simulator sickness level. The SSQ is well-established and has been widely used in many different simulator sickness studies (e.g. Cobb et al. (1999) and Häkkinen et al. (2006)).

The symptoms of motion and simulator sickness are similar to those observed during handheld projector interaction by Willis et al. (2011) and Kaufmann and Ahlström (2012). Therefore we believe, the SSQ is an appropriate method to measure symptoms of handheld projector sickness (HPS).

\section{EXPERIMENT}

We conducted a user study to find out if participants are experiencing HPS when watching handheld projector interaction. Observations during our pilot study indicated that HPS is more likely to occur when being an active observer, rather than being the operator of the handheld projector. Therefore, the experiment was designed so that all participants had to follow a demonstration of handheld projector interaction and to solve specific tasks that required their full attention during the demonstration. After the demonstration a questionnaire based on the Simulator Sickness Questionnaire was handed to the participants.

\subsection{Participants}

Twenty-six volunteers (twelve females) aged 10 to 51 years (mean 25.3, SD 10.3) participated. No participant had previous experience with interactive handheld projectors. None of them had a condition or an illness that would account for any symptoms while performing the task.

\subsection{Apparatus}

For the experiment we used an interactive handheld projector prototype that supports peephole interaction. In peephole interaction, a window (the peephole) displays only a portion of a much larger virtual workspace (Figure 1). When the peephole window is moved in space, by pointing with the projector along the projection wall, different areas of the virtual workspace can be accessed. Calculating the orientation of the projector allows for creating the illusion that the workspace is fixed to the projection wall. A good way to understand peephole interaction is to think about a dark room and a large picture hanging on the wall. By moving a flashlight across the wall, parts of the picture can be examined.

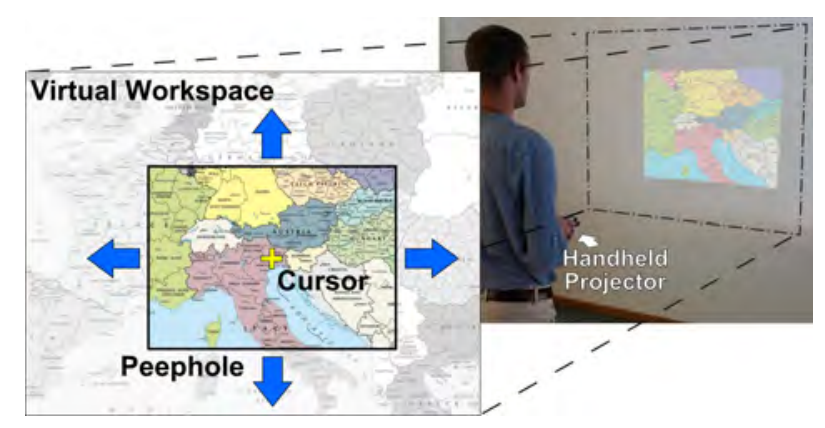

Figure 1: A window shows only a portion of a larger virtual workspace. Content outside the window is invisible. Moving the window reveals other parts of the workspace.

The interactive handheld projector prototype consists of three components: a smartphone (Samsung Nexus S running Android 2.3.4) calculating the orientation of the handheld device using its inertial sensors, a handheld projector (MicroVision SHOWWX+) that is attached to the smartphone, and a notebook computer rendering the content that is displayed with the projector. The projector is connected via HDMI to the Mini DisplayPort of the notebook computer. The smartphone maintains a WiFi connection to the notebook to transmit orientation data every $20 \mathrm{~ms}$.

\subsection{Setup and Procedure}

The projection surface (white wall) was 4.5 meters wide and 2.5 meters high and corresponded with the virtual workspace size. The experimenter who operated the handheld projector stood two meters in front of the projection wall. As a result, the peephole display dimensions were about one by one meter. All participants sat at tables three to four meters from the projection surface (Figure 2). 


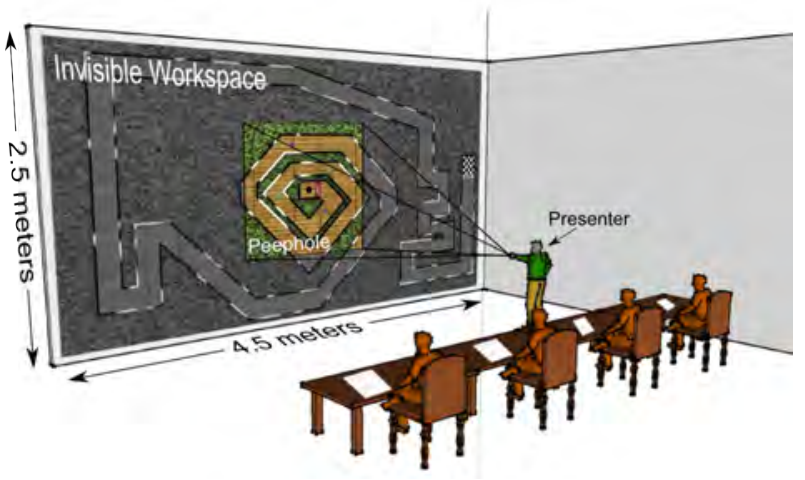

Figure 2: The presenter moves the peephole display across the virtual workspace following a race track (Task 1 to 3). Participants have to watch carefully to solve the given tasks. Content outside the peephole is invisible.

The experiment consisted of three sessions where we tested twenty-six participants in total. Participants were almost equally distributed among sessions. The room was darkened (i.e. blinds shut) with just as much light as necessary for participants to note down their results. Each session started at 5 p.m., had the same setup and followed the same procedure. Since previous studies taught us that at least ten to twenty minutes are necessary to identify simulator sickness symptoms (Strauss (2005)), we designed the test to last for at least half an hour.

Young et al. (2007) proved that participants who know that simulator sickness is being tested in an experiment will show higher indications of simulator sickness than those who do not know about the real intention of a simulator sickness test. As suggested by Young et al. (2007), we did not inform our participants about the full purpose of our study, instead they were told the goal of the study is to test the acceptance of a new interactive mobile computing device. Young et al. further recommend screening participants for absence of any illness that might bias the results. In the post-questionnaire all participants stated that they were in good health when the experiment started.

The test can be divided into seven parts:

- Demonstration of peephole interaction

- Task 1: Count red squares painted on the track

- Task 2: Solve equations shown next to the track

- Task 3: Count green crosses along the track

- Task 4: Count successfully selected items

- Task 5: Watch the presenter completing a test

- Fill out the questionnaires
At the beginning, instructions were given to the participants describing the tasks that had to be completed during the thirty minutes demonstration of the prototype. We asked the participants to stay as focused on the moving peephole display as possible in order to solve the given tasks. In addition, they were instructed not to talk or help each other with the given tasks. After the briefing, the concept of peephole interaction with a handheld projector was explained and demonstrated by showing a world map with the peephole device (illustrated in Figure 1).

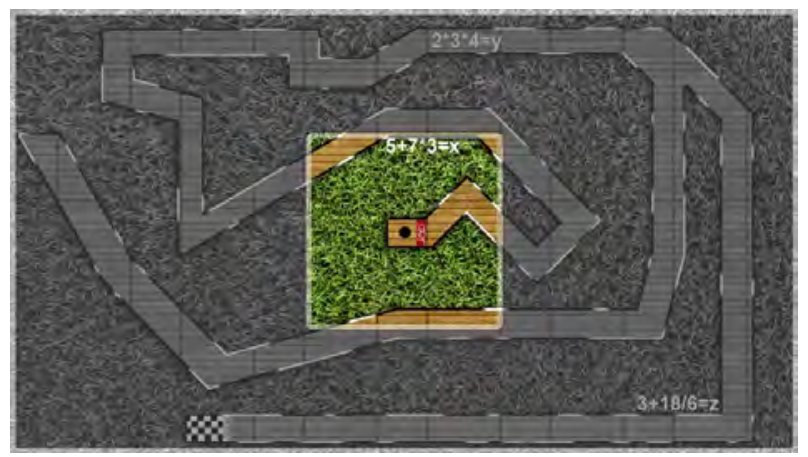

Figure 3: Race track from Task 2 with the peephole placed over the start position and an equation visible at the top of the peephole display.

In Task 1, 2 and 3 the presenter moved the peephole display slowly to follow different race tracks while trying to keep the cursor (a black dot centered within the peephole) on the track (Figure 3).

A task started after the previous one was completed. For each task a different race track was used. In Task 1 participants had to count red squares painted on the track at various places. Task 2 required participants to find and solve simple equations that appeared next to the track as pictured in Figure 3. In Task 3, they had to count green crosses placed along the track. These tasks were easy to complete, but forced the participants to constantly look at the screen. After each task, participants were asked to note down their results. Tasks 1 to 3 were performed twice before Task 4 was started.

In Task 4, green and blue squares appeared at random locations on a virtual workspace with a dark gray background. The presenter had to search for the squares by moving the peephole across the workspace. Once a square was found, it had to be selected with the cursor that is fixed at the center of the peephole (Figure 4).

When a square was selected, it disappeared and another square appeared at a randomized location. The workspace contained two squares at any time. In order to increase the challenge, the peephole size changed after every three correctly selected squares. The picture of Figure 4 shows a narrow band as a 


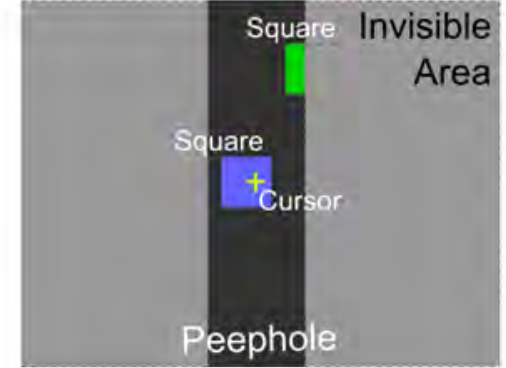

Figure 4: One partly-visible and one fully-revealed target from Task 4. Participants had to count successful selected targets.

peephole. In Task 4, participants had to count how many green and blue squares were selected.

Task 5 only required the participants to watch the presenter completing another round of selecting green and blue squares, but this time without counting the number of squares. The participants just had to focus on the screen. In Task 4 and 5, the peephole was moved rapidly, since the presenter tried to find and select the targets as fast as possible.

At the end, all participants were asked to fill out the questionnaire. The questionnaire contained both general open questions about the device (e.g. would you purchase such a device, how much would you pay for it, etc.) and the Simulator Sickness Questionnaire with its 4-point-scale (none, slight, moderate, severe) for each question.

\subsection{Results}

We analyzed the sixteen questions of the Simulator Sickness Questionnaire (SSQ) in order to find out if HPS was present when participants performed a series of tasks while watching handheld projector interaction. As suggested by Kennedy et al. (1993), we calculated the scores for the three sub scales, Nausea (N), Oculomotor (O) and Disorientation (D). Based on these three values the Total Severity (TS) score was derived.

Table 1 displays the resulting scores. Table 2 is provided as a reference indicating potential score ranges. For example, when all participants would have rated symptoms related to nausea as 'moderate', the resulting $\mathrm{N}$ score would have been 133.6 as shown in Table 2.

Table 1: SSQ Results

\begin{tabular}{|l|c|c|c|c|}
\hline & Nausea & Oculomotor & Disorientation & TS \\
\hline mean & 12.8 & 22.2 & 21.4 & 21.7 \\
\hline SD & 12.4 & 15.0 & 20.2 & 15.6 \\
\hline min & 0 & 0 & 0 & 0 \\
\hline max & 47.7 & 60.6 & 83.5 & 63.6 \\
\hline
\end{tabular}

Comparing the results of our study to the possible score ranges suggests that HPS is almost not present when observing handheld projector interaction. Although, the maximum scores indicate that in rare cases spectators of handheld projector interaction might feel slight symptoms. In fact, four out of twentysix participants had a Total Severity score above 41.1.

Table 2: Possible score ranges

\begin{tabular}{|l|c|c|c|c|}
\hline & Nausea & Oculomotor & Disorientation & TS \\
\hline none & 0 & 0 & 0 & 0 \\
\hline slight & 66.8 & 53.1 & 97.4 & 78.5 \\
\hline moderate & 133.6 & 106.1 & 194.9 & 157.1 \\
\hline severe & 200.3 & 159.2 & 292.3 & 235.6 \\
\hline
\end{tabular}

Females showed a slightly higher Oculomotor score (26.5 vs. 18.4) than males. Statistical analysis did not indicate further significant effects between scores for gender, age or computer gaming experience.

Kennedy et al. (1993) analyzed 1099 samples from participants using flight simulators and measured a mean TS score of 9.8 (SD 15.0) which is slightly lower that the 21.72 (SD 15.59) score we calculated. However, the SSQ inventors argue that the scale is not intended to differentiate between simulators with low scores, but rather should be used to distinguish between problematic simulators (high score) and unproblematic simulators (low score). Consequently, we would argue that our results show that HPS is unproblematic but mild symptoms might occur in sporadic situations.

\section{DISCUSSION}

We argue that handheld projector sickness is innocuous among active observers, however, under specific conditions the severity level might be higher. A larger field of view (FOV) or absence of reference points potentially affect the amount of dizziness. The FOV in our experiment was comparable to the FOV reported in Willis et al. (2011) and Kaufmann and Ahlström (2012); a size likely to occur in handheld projector interaction. Nevertheless, if an observer stands very close to the projection wall (e.g. at less than one meter distance to the wall), the FOV would have been much larger and at the same time the amount of reference points would have been lower. A situation that probably leads to different results. Therefore, varying the FOV (i.e. distance to the projection) might be worthwhile to address in future studies.

Although, often people might stand while watching handheld projector interaction, we decided to let participants sit. Regan and Price (1993) conducted a study with 44 participants to find out if standing or sitting causes more sickness while 
navigating through a virtual environment. They found no significant difference between these two conditions but highlighted that sitting participants rated moderate and severe nausea higher compared to the standing group.

After the experiment, most of the participants wanted to try the handheld projector on their own. According to their informal comments they found the presented interactive peephole pointing device intuitive, exciting and fun to play with. However, one participant mentioned that she felt sicker when using the device herself. On the other hand, she was already informed about the real intention of the study and knew that we were studying HPS. Thus, she could have been biased as highlighted by Young et al. (2007). Previous user studies about handheld projector interaction never mentioned motion or simulator sickness symptoms when using an interactive handheld projector as a user, and drawn from our own experience we never observed such symptoms either besides the comment described above.

\section{CONCLUSION AND FUTURE WORK}

We presented a study that evaluated the occurrence of handheld projector sickness (HPS) among spectators of handheld projector interaction. The results suggest that active observers of handheld projector interaction will not be at risk of HPS, but they leave open the question of effects on other participants in the interaction. Future work should include an examination of the SSQ ratings of passive observers and of actual users of the projector.

It would also be interesting to see if stimuli related to motion sickness might contribute to symptom presentation, as in the case of shared projector interaction that might take place between passengers in a moving commuter vehicle. Finally, long term studies should be pursued in order to measure either cumulative sensitivity or cumulative resistance over time. In all of these cases, it might be informative to add physiological measures of stress and nausea to the experimental design.

\section{REFERENCES}

Beardsley, P., van Baar, J., Raskar, R., and Forlines, C. (2005) Interaction using a handheld projector. IEEE Computer Graphics and Applications, 25(1), 39-43.

Cao, X., Forlines, C., and Balakrishnan, R. (2007) Multi-user interaction using handheld projectors. In Proceedings of UIST'07, Newport, Rhode Island, USA, pp. 43-52, ACM, New York, NY, USA.
Cobb, S. V. G., Nichols, S., Ramsey, A., and Wilson, J. R. (1999) Virtual reality-induced symptoms and effects (VRISE). Presence, 8(2), 169-186.

Häkkinen, J., Pölönen, M., Takatalo, J., and Nyman, G. Simulator sickness in virtual display gaming: a comparison of stereoscopic and non-stereoscopic situations. In Proceedings of MobileHCl'06, Helsinki, Finland, pp. 227-230, ACM, New York, NY, USA.

Kaufmann, B., and Ahlström, D. (2012) Revisiting Peephole Pointing: A Study of Target Acquisition with a Handheld Projector. In Proceedings of MobileHCl'12, San Francisco, CA, USA, ACM, New York, NY, USA.

Kennedy, R. S., Lane, N. E., Berbaum, K. S., and Lilienthal, M. G. (1993) Simulator sickness questionnaire: an enhanced method for quantifying simulator sickness. International Journal of Aviation Psychology, 3, 203-220.

NAVTRADEBCEN 1915-00-1 (1957) Evaluation of training effectiveness of the $2 \mathrm{FH} 2$ helicopter flight trainer research tool., Naval Training Device Center, Port Washington, NY, USA.

Regan, E. C., and Price, K. R. (1993) Some sideeffects of immersion virtual reality: the effect of increasing head movements, of rapid interaction, and of seating subjects. Army Personnel Research Establishment, Report 93R022, Farnborough, U.K.

Strauss, S. H. (2005) New, improved, comprehensive, and automated driver's license test and vision screening system. Arizona Department of Transportation. http://www.azdot.gov/tpd/atrc/publications/project_ reports/pdf/az559.pdf (12 June 2012)

Willis, K. D. D., Poupyrev, I., Hudson, S. E., and Mahler, M. (2011) SideBySide: Ad-hoc multi-user interaction with handheld projectors. In Proceedings of UIST'11, Santa Barbara, CA, USA, pp. 431-440, ACM, New York, NY, USA.

Willis, K. D. D., Poupyrev, I., and Shiratori, T. (2011) Motionbeam: a metaphor for character interaction with handheld projectors. In Proceedings of $\mathrm{CHI}$ '11, Vancouver, BC, Canada, pp. 1031-1040, ACM, New York, NY, USA.

Winkler, C., Reinartz, C., Nowacka, D., and Rukzio, E. (2011) Interactive phone call: synchronous remote collaboration and projected interactive surfaces. In Proceedings of ITS'11, Kobe, Japan, pp. 61-70, ACM, New York, NY, USA.

Young, S. D., Adelstein, B. D., and Ellis, S. R. (2007) Demand characteristics in assessing motion sickness in a virtual environment: or does taking a motion sickness questionnaire make you sick? IEEE Transaction on Visualization and Computer Graphics, 13, $422-428$ 